\title{
Distinctive properties of plaque-type dura mater graft-associated Creutzfeldt-Jakob disease in cell-protein misfolding cyclic amplification
}

\author{
Atsuko Takeuchi ${ }^{1}$, Atsushi Kobayashi ${ }^{2}$, Piero Parchi ${ }^{3,4}$, Masahito Yamada ${ }^{5}$, Masanori Morita ${ }^{6}$, Shusei Uno ${ }^{6}$ and \\ Tetsuyuki Kitamoto ${ }^{1}$
}

There are two distinct subtypes of dura mater graft-associated Creutzfeldt-Jakob disease (dCJD) with methionine homozygosity at codon 129 of the PRNP gene. The majority of cases is represented by a non-plaque-type (np-dCJD) resembling sporadic CJD (sCJD)-MM1 or -MV1, while the minority by a plaque-type (p-dCJD). p-dCJD shows distinctive phenotypic features, namely numerous kuru plaques and an abnormal isoform of prion protein ( $\mathrm{Pr} \mathrm{P}^{\mathrm{SC}}$ ) intermediate in size between types 1 and 2. Transmission studies have shown that the unusual phenotypic features of $\mathrm{p}$-dCJD are linked to the V2 prion strain that is associated with SCJD subtypes VV2 or -MV2. In this study, we applied protein misfolding cyclic amplification (PMCA) using recombinant human prion protein as a substrate and demonstrated that $\mathrm{p}$-dCJD prions show amplification features that are distinct from those of $n p-d C J D$. Although no amplification of $n p-d C J D$ prions was observed with either $129 \mathrm{M}$ or $129 \mathrm{~V}$ substrate, $\mathrm{p}$-dCJD prions were drastically amplified with the $129 \mathrm{~V}$ substrates, despite the PRNP codon 129 incompatibility between seed and substrate. Moreover, by using a type $2 \mathrm{PrP}^{\mathrm{SC}}$-specific antibody not recognizing $\mathrm{PrP}^{\mathrm{Sc}}$ in $\mathrm{p}-\mathrm{dCJD}$, we found that type 2 products are generated de novo from $\mathrm{p}-\mathrm{dCJD}$ prions during PMCA with the $129 \mathrm{~V}$ substrates. These findings suggest that our cell-PMCA is a useful tool for easily and rapidly identifying acquired CJD associated with the transmission of the V2 CJD strain to codon 129 methionine homozygotes, based on the preference for the $129 \mathrm{~V}$ substrate and the type of the amplified products.

Laboratory Investigation (2016) 96, 581-587; doi:10.1038/labinvest.2016.27; published online 15 February 2016

Creutzfeldt-Jakob disease (CJD) is a fatal neurodegenerative disease. The large majority of CJD cases are thought to be caused by a spontaneous conformational change of the normal monomeric isoform of the prion protein $\left(\mathrm{PrP}^{\mathrm{C}}\right)$ into an abnormal isoform $\left(\mathrm{PrP}^{\mathrm{Sc}}\right)$, as in sporadic CJD (sCJD) or genetic/familial CJD. On the other hand CJD can also be acquired through $\mathrm{PrP}^{\mathrm{Sc}}$ infection, as in variant $\mathrm{CJD}$ or iatrogenic CJD (iCJD). The wide heterogeneity of sCJD clinico-pathological phenotypes depends on both the genotype (methionine $(\mathrm{M})$ or valine $(\mathrm{V})$ ) at the polymorphic codon 129 of the PRNP gene and the type (1 or 2 ) of $\mathrm{PrP}^{\mathrm{Sc}}$ accumulating in the brain. ${ }^{1}$ Type 1 and type 2 can be distinguished according to the size of the proteinase K-resistant core of the protein (21 and $19 \mathrm{kDa}$, respectively) on western blots. Based on the polymorphism at codon 129 of the PRNP gene and the type of $\mathrm{PrP}^{\mathrm{Sc}}$, sCJD patients are classified into six major subtypes: MM1/MV1, MM2 cortical, MM2 thalamic, VV1, VV2, and MV2. ${ }^{1}$

iCJD is caused by the transmission of prions via cadaveric pituitary hormones, dura mater, and corneal grafts, or contaminated neurosurgical instruments. Many cases of dura mater graft-associated CJD (dCJD) have been reported in Japan..$^{2-4}$ There are two distinct phenotypes in dCJD with methionine homozygosity at codon 129 . The first is a major group represented by a non-plaque-type dCJD (np-dCJD), the second a minor group represented by a plaque-type dCJD $(\mathrm{p}-\mathrm{dCJD}) .^{5-7} \mathrm{np}$-dCJD shares phenotypic characteristics such as diffuse synaptic-PrP deposition and type $1 \mathrm{PrP}^{\mathrm{Sc}}$ with sCJD-MM1 or -MV1, the most common sCJD phenotype (denoted as M1 strain in transmission studies). ${ }^{4,8}$ In contrast,

${ }^{1}$ Department of Neurological Science, Tohoku University Graduate School of Medicine, Sendai, Japan; ${ }^{2}$ Laboratory of Comparative Pathology, Hokkaido University, Graduate School of Veterinary Medicine, Sapporo, Japan; ${ }^{3}$ RCCS, Istituto delle Scienze Neurologiche, Bologna, Italy; ${ }^{4}$ Department of Biomedical and Neuromotor Sciences, University of Bologna, Bologna, Italy; ${ }^{5}$ Department of Neurology and Neurobiology of Aging, Kanazawa University Graduate School of Medical Science, Kanazawa, Japan and ${ }^{6}$ Research and Development Division, Japan Blood Products Organization, Tokyo, Japan

Correspondence: Professor T Kitamoto, Department of Neurological Science, Tohoku University Graduate School of Medicine, 2-1, Seiryo-machi, Aoba-ku, Sendai 9830036, Japan.

E-mail: kitamoto@med.tohoku.ac.jp

Received 11 September 2015; revised 10 December 2015; accepted 4 January 2016 
$\mathrm{p}$-dCJD is characterized by unusual phenotypic features such as the presence of numerous kuru plaques and a unique $\operatorname{PrP}^{\mathrm{Sc}}$ type with an electrophoretic mobility of about $20 \mathrm{kDa}$, which is intermediate in size between types 1 and $2\left(\right.$ type i $\left.\operatorname{PrP}^{\mathrm{Sc}}\right) .{ }^{9}$

We previously demonstrated that the transmission properties of p-dCJD prions resemble those of sCJD-VV2 or -MV2 prions and, in particular, that the transmission of sCJD-VV2 prions to humanized knock-in mice carrying the $129 \mathrm{MM}$ genotype produce type i $\operatorname{Pr}^{S c}$ and kuru plaques, indicating that this subtype of dCJD is caused by cross-sequential transmission of sCJD-VV2 or -MV2 (denoted as V2 strain), ${ }^{8}$ the second most common CJD strain, to individuals with codon 129 methionine homozygosity. ${ }^{9-11}$ Interestingly, type i $\operatorname{PrP}^{\mathrm{Sc}}$ and kuru plaques in patients homozygous for methionine at codon 129 have only been observed in acquired prion diseases, especially in p-dCJD. ${ }^{9,12-15}$

A correct diagnosis and classification of cases is of critical importance for CJD surveillance centers worldwide aiming to identify potentially new disease forms or risk factors. Although $\operatorname{PrP}^{\mathrm{Sc}}$ typing may be considered a tool for identification of the CJD etiology (eg, sporadic vs acquired) when no reliable evidence of CJD infection is available from clinical records, it is rather difficult to differentiate type i from type 1 $\mathrm{PrP}^{\mathrm{Sc}}$ in routine western blot analysis due to the subtle differences in their electrophoretic mobility. In a transmission study with knock-in humanized mice, we have recently demonstrated, for example, that two cases with atypical CJD$\mathrm{MM}$ with plaques, reported as SCJD in the literature, were actually acquired CJD cases caused by the V2 strain. ${ }^{16}$ Therefore, the most reliable current approach to identify acquired CJD is to carry out expensive and time-consuming experimental transmission studies. Under these circumstances, this study was undertaken to establish a new, rapid, and reliable method for the differentiation of acquired CJD from SCJD with PMCA using cell lysates containing exogenously expressed human $\operatorname{PrP}^{\mathrm{C}}$ as a substrate (denoted as cell-PMCA). ${ }^{17} \mathrm{We}$ investigated the relative amplification efficiency of $\operatorname{PrP}^{\mathrm{Sc}}$ and characterized $\operatorname{PrP}^{\mathrm{Sc}}$ amplified products in patients with p-dCJD, np-dCJD, and sCJD. Based on the results obtained, we propose that p-dCJD (V2 strain) can be reliably distinguished from np-dCJD (M1 strain) according to distinctive amplification properties.

\section{MATERIALS AND METHODS}

\section{Ethic Statement and Brain Tissues}

Brain tissues were obtained at autopsy after receiving written informed consent for research use. For each selected case, the results of $\operatorname{PrP}^{\mathrm{Sc}}$ immunohistochemistry, PRNP sequence analysis, and $\operatorname{PrP}^{\mathrm{Sc}}$ typing by western blotting were available in addition to clinical and histopathological data. ${ }^{18-20}$ Brain homogenates were prepared from dCJD (np-dCJD or p-dCJD) cases with MM at codon 129, five different sCJD subtypes (MM1, MM2, MV1, MV2, or VV2), two atypical CJD-MM cases with plaques, and nine non CJD controls. Atypical CJD case \#1 was a patient who had a medical history of neurosurgery without dura mater grafting, and case \#2 was a neurosurgeon. Details about the clinico-pathological and molecular features, including transmission properties of the two atypical sCJD-MM cases with plaques were previously described. ${ }^{16,21-23}$ Brains were homogenized at $10 \%(\mathrm{w} / \mathrm{v})$ in PMCA buffer (50 mM Phosphate buffer (pH 6.8) containing $4 \mathrm{mM}$ ethylenediaminetetraacetic acid (EDTA), $80 \mathrm{mM} \mathrm{NaCl}$, $1.0 \%$ Nonidet P-40, and Complete Protease Inhibitor Cocktail (Roche)) and stored at $-80^{\circ} \mathrm{C}$ in small aliquots.

\section{Preparation of Substrates}

FreeStyle 293F cell (Invitrogen) lysates were used as substrate for cell-PMCA of human prions. Twenty percent $(\mathrm{w} / \mathrm{v})$ lysates from a cell line stably expressing human $\operatorname{PrP}^{\mathrm{C}}$ with methionine at codon 129 were prepared as described previously, ${ }^{17}$ with some modifications. Cells were homogenized at $20 \%(\mathrm{w} / \mathrm{v})$ in PMCA buffer and stored at $-80^{\circ} \mathrm{C}$ in small aliquots. For cells expressing human $\operatorname{PrP}^{\mathrm{C}}$ with valine at codon 129, a cDNA for chimeric mouse-human PrP with $129 \mathrm{~V}$ was constructed as described. ${ }^{24}$ Since $293 \mathrm{~F}$ cells endogenously express detectable levels of human $129 \mathrm{MPrP}^{\mathrm{C}}$, to minimize the effects of simultaneous expression of both $129 \mathrm{MPrP}^{\mathrm{C}}$ and $129 \mathrm{VPrP}^{\mathrm{C}}$, we prepared a human $129 \mathrm{MPrP}^{\mathrm{C}}$ cell line by RNA interference-mediated knockdown using short hairpin RNA (shRNA). To target the $5^{\prime}$ untranslated region of human PRNP mRNA (5'-GGACTTAGTGCAA CAGGTT-3'), a shRNA expression vector was designed. A double-stranded DNA oligonucleotide encoding the chosen shRNA with the sense (5'-GATCCGGACTTAGTGCAACAC CTTTAGTGCTCCTGGTTGAACCTGTTGCACTAAGTCCT TTTTTA- $3^{\prime}$ ) and antisense sequences (5'-AGCTTAAAAAA GGACTTAGTGCAACAGGTTCAACCAGGAGCACTAAACC TGTTGCACTAAGTCCG - $3^{\prime}$ ) was cloned into the expression vector pBAsi-hUp pur (Takara bio Inc, Japan) after digestion with BamHI and XhoI. The generated plasmids were linearized with ScaI (Takara) and transfected into 293F cells using Lipofectamine 2000 transfection reagent (Invitrogen), according to the manufacturer's instruction. Cells expressing the lowest levels of PRNP mRNA were selected by real-time PCR, and maintained in Freestyle293 expression medium (Gibco) supplemented with puromycin. The levels of endogenous expression of either PRNP mRNA or $\mathrm{PrP}^{\mathrm{C}}$ in the knockdown cells were reduced by $90 \%$ or $95 \%$, respectively. Twenty percent $(\mathrm{w} / \mathrm{v})$ lysates of $129 \mathrm{MPrP} \mathrm{P}^{\mathrm{C}}$ knockdown cell line stably expressing human $129 \mathrm{~V}-\operatorname{PrP}^{\mathrm{C}}$ were prepared as described above. Human $129 \mathrm{M}$ - or $129 \mathrm{~V}$ - $\operatorname{PrP}^{\mathrm{C}}$ concentration in the each of the two $20 \%(\mathrm{w} / \mathrm{v})$ cell lysates was $5-10$ times higher than the concentration of human $\mathrm{PrP}^{\mathrm{C}}$ in $10 \%$ $(\mathrm{w} / \mathrm{v})$ brain homogenates from knock-in humanized mice. Cell lysates were stored at $-80^{\circ} \mathrm{C}$ in small aliquots until use.

\section{Estimation of PrP ${ }^{\mathrm{Sc}}$ Levels in Brain Homogenates}

After partial purification with collagenase digestion and Sarkosyl- $\mathrm{NaCl}$ extraction, samples were run in $12 \%$ Mini-PROTEAN TGX Precast Gels (Bio-Rad Laboratories), 
together with an internal positive control with optimal signal intensity, and probed with the primary antibody 3F4 (Signet). ${ }^{25}$ Undiluted and 10 -fold diluted brain samples were loaded together with $10 \mu \mathrm{l}$ of the control. Western blot signal intensities were measured by densitometry using the Quantity One software and the imaging device VersaDoc 5000 (Bio-Rad Laboratories). The relative concentration of $\operatorname{PrP}^{\text {res }}$ in each brain homogenate was expressed by the dilution factor needed to equalize the signal intensity of $\operatorname{PrP}^{\text {res }}$ between the examined samples and the positive control.

\section{Cell-PMCA}

Cell-PMCA was performed as previously described, ${ }^{17}$ with some modifications. For amplification of CJD prions, brain homogenates were combined with the cell lysates, expressing human $\operatorname{PrP}^{\mathrm{C}}$ carrying either $\mathrm{M}$ or $\mathrm{V}$ at codon 129 , in a 0.1-ml-thin-walled PCR tube. Mixtures were then subjected to rounds of PMCA up to a $10^{-4}$ fold dilution (eg, 100, 1000, 10.000 times) using the cell lysate as the diluent. Since the amount of $\operatorname{PrP}^{\mathrm{Sc}}$ accumulated in each brain was extremely variable, brain homogenates were subjected to PMCA reaction after serial dilution in cell lysates without adjusting the amounts of the $\operatorname{PrP}^{\mathrm{Sc}}$ seed. Each PMCA round comprised 48 cycles of sonication (5 pulses of $5 \mathrm{~s}$ with 1-s rest) and agitation $\left(1 \mathrm{~h}\right.$ at $\left.37^{\circ} \mathrm{C}\right)$ and was carried out using a fully automatic cross-ultrasonic protein activating apparatus (ELESTEIN 070-GOT, Elekon Science, Japan).

\section{Proteinase K Digestion and Western Blotting Analysis}

Either before or after PMCA, samples were digested with $50 \mu \mathrm{g} / \mathrm{ml}$ proteinase $\mathrm{K}$ at $37^{\circ} \mathrm{C}$ for $60 \mathrm{~min}$. The digested samples were subjected to SDS-PAGE and western blot analysis. Anti-PrP monoclonal antibody $3 \mathrm{~F} 4$ or type $2 \mathrm{PrP}^{\mathrm{Sc}}{ }_{-}$ specific polyclonal antibody (designated as Tohoku $2(\mathrm{~T} 2))^{10}$ was used as primary antibodies. Anti-rabbit EnVision+ or anti-mouse EnVision+ (Dako) were used as secondary antibodies. Enhanced chemiluminescence (GE Healthcare) was used to visualize the immunoreactivity. Signal intensities at the optimal exposure time were quantified by densitometry using a CCD camera and the imaging device VersaDoc 5000. Amplification factors were obtained by measuring western blot signals of amplified $\mathrm{PrP}^{\mathrm{Sc}} /$ seeded $\mathrm{PrP}^{\mathrm{Sc}}$.

\section{RESULTS}

Western blot profiles of $\operatorname{PrP}^{\text {res }}$ from representative CJD brain homogenates that were used as seeds for cell-PMCA are shown in Figure 1. It was difficult to recognize subtle mobility differences in the non-glycosylated forms between type 1- and type i-PrP $\mathrm{P}^{\text {res }}$ using $15 \%$ SDS-PAGE mini gels (Figure 1, lane 3 and 6). Furthermore, as expected, $\operatorname{PrP}^{\mathrm{Sc}}$ type 2-specific antibody (T2) revealed a specific signal in both sCJD-VV2 and -MV2 cases (Figure 1, lane 4 and 8), while it did not recognize $\mathrm{PrP}^{\mathrm{Sc}}$ in both np-dCJD and p-dCJD (Figure 1, lane 2, 3, 6 and 7).

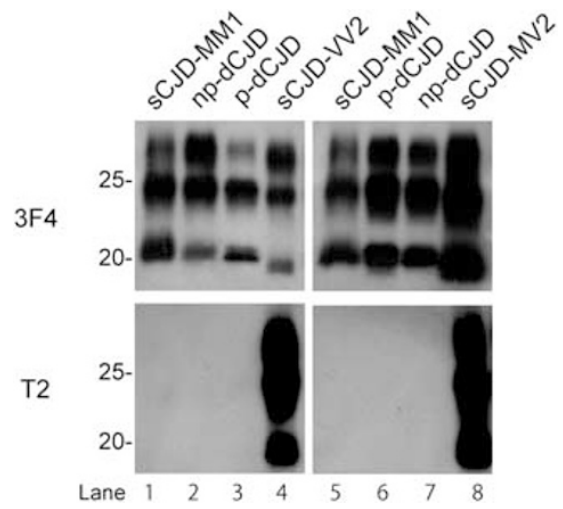

Figure 1 Western blot analysis of PrPres in the brain homogenate seeded for cell-PMCA. Ten percent ( $w / v)$ CJD brain homogenates were digested with proteinase $\mathrm{K}\left(50 \mu \mathrm{g} / \mathrm{ml}\right.$ at $37^{\circ} \mathrm{C}$ for $\left.60 \mathrm{~min}\right)$. Anti-PrP monoclonal antibody $3 \mathrm{~F} 4$ (upper panel) or type $2 \mathrm{PrP}^{\mathrm{Sc}}$-specific polyclonal antibody (T2) (lower panel) was used as the primary antibody to detect PrPres. Numbers show the molecular size standards (kDa). CJD, Creutzfeldt-Jakob disease; np-dCJD, non-plaque-type CJD; p-dCJD, plaque-type CJD; PMCA, protein misfolding cyclic amplification; SCJD, sporadic CJD.

We applied cell-PMCA using recombinant human $129 \mathrm{MPrP}^{\mathrm{C}}$ or $129 \mathrm{VPrP}^{\mathrm{C}}$ as a substrate to compare the relative amplification efficiency of $\mathrm{PrP}^{\mathrm{Sc}}$ among $114 \mathrm{CJD}$ patients. The calculated amplification factors for each CJD case are summarized in Table 1 and Supplementary Table 1, and representative western blots are shown in Figure 2. No significant signal of the amplified products was observed using either sCJD-MM1 or -MM2 prions as seed, irrespective of whether the genotype at codon 129 of the substrate was M or V (Figure 2a, upper panel and Supplementary Table 1). In contrast, $\mathrm{PrP}^{\mathrm{Sc}}$ signals from $\mathrm{sCJD}-\mathrm{VV} 2$ and -MV2 prions were significantly amplified, up to a $10^{-3}$-folds dilution, although only with the $129 \mathrm{~V}$ substrate (Figure 2e, upper panels). Overall, these results demonstrate that, in the tested PMCA conditions, the $\mathrm{V} 2$ strain is amplified more efficiently than the M1 or M2 strains, and that the valine genotype at codon 129 makes the substrate more susceptible to conversion by the V2 strain.

Likewise no efficient amplification of the sCJD-MM1 prions was observed in the nine cases of np-dCJD regardless of whether the codon 129 genotype of substrate was M or V, suggesting that the seeding activity of $\mathrm{np}-\mathrm{dCJD}$ prions matches that of sCJD-MM1 prions (Figure 2a, upper panel). In contrast, $\mathrm{PrP}^{\mathrm{Sc}}$ from $\mathrm{p}$-dCJD cases was strongly amplified in the presence of $129 \mathrm{~V}$ substrate despite the mismatched codon 129 genotype between the seed and substrate (Figure 2c, upper panel), indicating that $\mathrm{p}$-dCJD prions, like sCJD-VV2 or -MV2 prions, have a clear preference for the $129 \mathrm{~V}$ genotype.

There was a significant variability in the amplification factor among p-dCJD cases (Table 1), which might reflect an heterogeneity in the $\operatorname{PrP}^{\mathrm{Sc}}$ content among brain homogenates used as seed. Amplification products were detected up to a $10^{-3}$-fold dilution in all cases, and up to a $10^{-4}$-fold dilution 


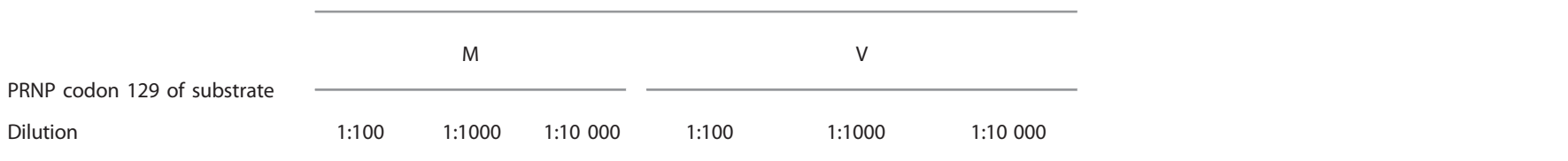

$\operatorname{CJD}(\mathrm{n}=114)$

SCJD

$\operatorname{MM1}(n=77)$

MM2 $(n=10)$

$\operatorname{MV1}(n=1)$

$\operatorname{MV} 2 \mathrm{~K}(n=3)$

MV2 case \#1

MV2 case \#2

MV2 case \#3

W2 $(n=1)$

$d C J D$

$n p-d C J D(n=9)$

np-dCJD case \#1

np-dCJD case \#2

$\mathrm{np}$-dCJD case \#3

$\mathrm{np}$-dCJD case \#4

$\mathrm{np}$-dCJD case \#5

np-dCJD case \#6

np-dCJD case \#7

$\mathrm{np}$-dCJD case \#8

np-dCJD case \#9

$p-d C J D(n=11)$

p-dCJD case \#1

p-dCJD case \#2

p-dCJD case \#3

p-dCJD case \#4

p-dCJD case \#5

p-dCJD case \#6

p-dCJD case \#7

p-dCJD case \#8

p-dCJD case \#9

p-dCJD case \#10

p-dCJD case \#11
$<2$

$<2$

$<2$

$<2$

$<2$

$<2$

$2.6( \pm 0.1)$

$6.7( \pm 6.5)$

$3.5( \pm 0.4)$

$<2$

$<2$

$<2$

$2.1( \pm 0.3)$

$<2$

$47.6( \pm 2.2)$

$42.0( \pm 29.5)$

$95.9( \pm 0.1)$

$45.6( \pm 8.6)$

$42.5( \pm 6.5)$

$<2$

$206.7( \pm 31.0)$
$<2$

$<2$

$<2$

$6.0( \pm 1.0)$

$<2$

$<2$
30

typical CJD-MM with plaques

\section{Case $\# 1^{\text {a }}$ \\ Case \#2}

Non CJD $(\mathrm{n}=9)$

$\begin{array}{ccc}<2 & <2 & <2 \\ <2 & <2 & <2 \\ <2 & <2 & <2 \\ <2 & <2 & <2 \\ <2 & <2 & <2 \\ 2.1( \pm 0.8) & <2 & <2 \\ <2 & <2 & <2 \\ <2 & <2 & <2 \\ <2 & <2 & <2\end{array}$

$<2$

$<2$

$$
<2
$$

$<2$

$<2$

$<2$

$<2$

$<2$

$4.3( \pm 3.1)$

$2.5( \pm 0.3)$

$<2$

$<2$

$<2$

$\begin{array}{ccc}<2 & <2 & <2 \\ <2 & <2 & <2 \\ <2 & 3.4(+0.9) & <2\end{array}$

$<2$

$19.1( \pm 2.7)$

$128.7( \pm 60.5)$

$71.9( \pm 47.5)$

$154.2( \pm 29.5)$

$20.2( \pm 26.7)$

$8.5( \pm 3.1)$

$57.0( \pm 18.8)$

$95.5( \pm 20.7)$

$35.3( \pm 15.4)$

$41.7( \pm 21.2)$

$18.0( \pm 1.2)$

$18.6( \pm 2.6)$

$9.1( \pm 8.7)$

$$
<2
$$

$<2$

$<2$

$<2$

$<2$

$<2$

$<2$

$<2$

$<2$

$\begin{array}{cc}174.9 & ( \pm 209.1) \\ <2 & 47 \\ 307.8( \pm 73.0) & 1 \\ <2 & 53 \\ <2 & 3 \\ 92.0( \pm 62.9) & 6 \\ <2 & 90 \\ 3.2( \pm 0.6) & 1 \\ 3.5( \pm 1.2) & 4 \\ <2 & 35 \\ <2 & 6 \\ <2 & 5\end{array}$

${ }^{a}$ Details of the atypical CJD-MM cases with plaques have been reported elsewhere. ${ }^{21-23}$

Mean values of amplification factor for sCJD-MV2, VV2, np-dCJD, or p-dCJD brain were obtained from experiments performed at least three times.

The amplification factors were obtained by amplified PrPSc/seeded PrPSc in western blots using anti-PrP monoclonal antibody 3 F4 as primary antibody.

No amplification or no detection of seeded PrPSc signals was represented by ' $<2$ '. 
a
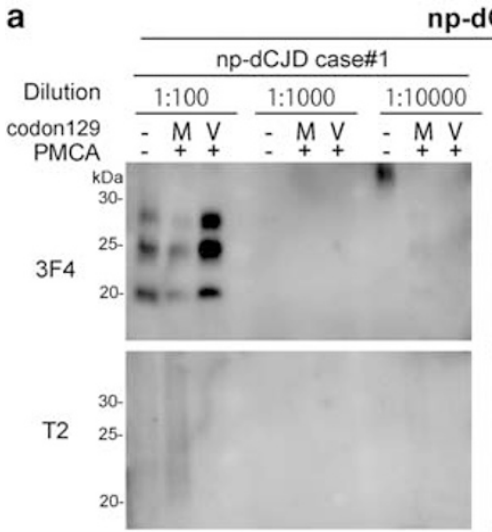

C

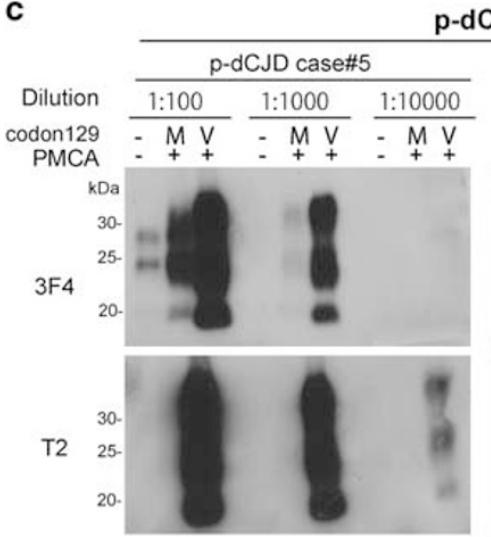

e

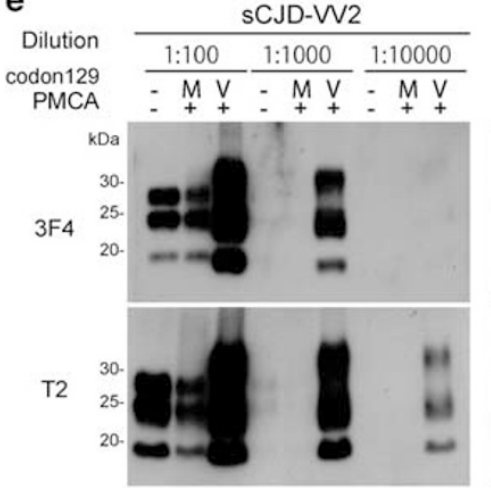

b
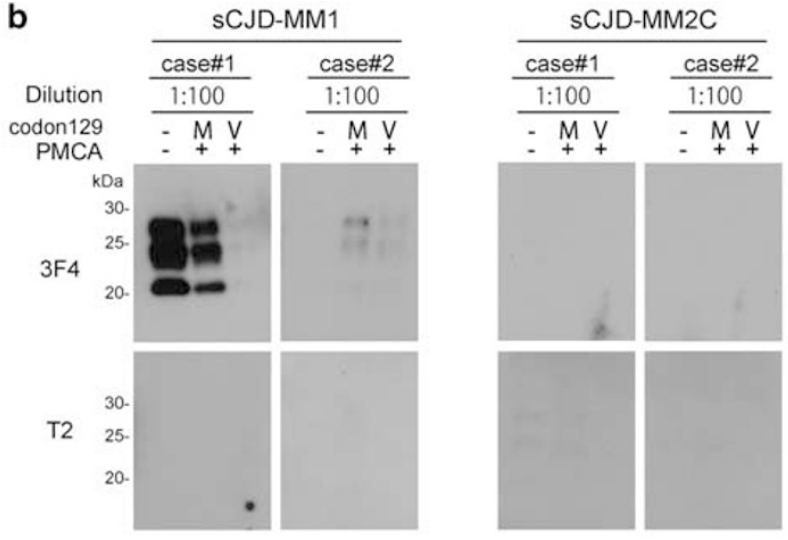

d

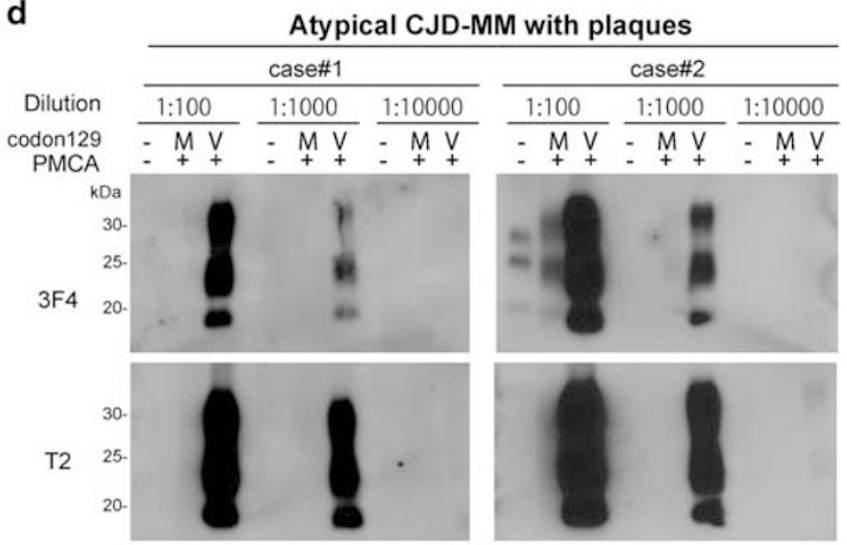

Figure 2 Western blot analysis of cell-PMCA products. PrP $\mathrm{P}^{\mathrm{Sc}}$ in the brain homogenates from patients with np-dCJD (a), sCJD-MM1 or -MM2C (b), p-dCJD (c), atypical CJD-MM with plaques (d) and SCJD-VV2 or -MV2K (e) were amplified with substrate lysates prepared from $293 \mathrm{~F}$ cells stably expressing human $129 \mathrm{MPrP}{ }^{C}$ or human $129 \mathrm{VPrP}^{\mathrm{C}}$. Ten-fold serial dilutions of CJD brain homogenates were seeded into the substrates, and aliquots were immediately stored at $-80^{\circ} \mathrm{C}$ (no PMCA) or subjected to 48 cycles of PMCA. Either before or after, the PMCA samples were treated with proteinase $\mathrm{K}$ $\left(50 \mu \mathrm{g} / \mathrm{ml}\right.$ at $37^{\circ} \mathrm{C}$ for $60 \mathrm{~min}$ ) and subjected to western blotting using the anti-PrP monoclonal antibody $3 \mathrm{~F} 4$ (upper panel) or the type 2 PrP ${ }^{\mathrm{SC}}$-specific polyclonal antibody T2 (lower panel). Signals with the T2 antibody in $10^{-2}$-fold dilutions of sCJD-VV2 or -MV2 prions in the 129M substrates (e, lower panel) might be the original type $2 \mathrm{PrP}^{\mathrm{SC}}$ in the brain homogenates seeded. Each of the results was typical of experiments performed at least two or three times. Numbers show the molecular size standards (kDa). CJD, Creutzfeldt-Jakob disease; np-dCJD, non-plaque-type CJD; p-dCJD, plaque-type CJD; PMCA, protein misfolding cyclic amplification; SCJD, sporadic CJD.

in some cases showing a relatively high $\mathrm{PrP}^{\mathrm{Sc}}$ content in the undiluted homogenate. In the latter group, the calculated amplification factors at a $10^{-2}$-fold dilution were likely overestimated due to the excess of $\mathrm{PrP}^{\mathrm{Sc}}$ in the seeded brain homogenate (Table 1, p-dCJD case \#3, \#6, or \#9).
As next step, we characterized the PMCA products using the T2 antibody. While no amplifications were detected with either sCJD-MM1, MM2, or np-dCJD prions (Figures 2a and b, lower panel), amplified products strongly reacting with $\mathrm{T} 2$ were detected in all sCJD-VV2 or -MV2 prions seeded with 
the $129 \mathrm{~V}$ substrate (Figure 2e, lower panel). Interestingly, a strong T2-reactive signal was also observed in all amplified products obtained using $\mathrm{p}-\mathrm{dCJD}$ as seed and the $129 \mathrm{~V}-\mathrm{PrP}$ as substrate, despite the original $\mathrm{PrP}^{\mathrm{Sc}}$ in $\mathrm{p}$-dCJD being not recognized by T2 (Figure 2c, lower panel). Furthermore, no T2-reactive PMCA product was detected with the $129 \mathrm{M}$ substrate. Taken together, these results clearly show that the amplification characteristics of p-dCJD prions fully differ from those of np-dCJD prions, and rather match those of sCJD-VV2 or -MV2 prions, ie, strong amplification with the $129 \mathrm{~V}$ substrate and the generation of type $2 \mathrm{PrP}^{\mathrm{Sc}}$ products.

We then analyzed the seeding activity and amplified products of two cases affected by atypical CJD-129MM with plaques. ${ }^{21-23}$ Both cases showed abundant kuru plaques and type $\mathrm{i} \operatorname{PrP}^{\mathrm{Sc}}$ and preferentially transmitted to humanized $129 \mathrm{~V} / \mathrm{V}$ mice resulting in the accumulation of type $2 \mathrm{PrP}^{\mathrm{Sc}}$ accumulation in the recipient animals. ${ }^{16}$ While the $\mathrm{PrP}^{\mathrm{Sc}}$ from both cases did not amplify at all with the $129 \mathrm{M}$ substrates despite the matched codon 129 genotypes, a very efficient amplification was seen with the $129 \mathrm{~V}$ substrate (Figure 2d, upper panel). Consistently, a strong $\operatorname{PrP}$ signal, also recognized by the $\mathrm{T} 2$ antibody (Figure $2 \mathrm{~d}$, lower panel), was detected up to a $10^{-3}$-fold dilution in both cases (Figure 2d, upper panel).

\section{DISCUSSION}

We have demonstrated that $\mathrm{p}$-dCJD prions differ from $n p-d C J D$ prions in their PMCA properties, namely the preference for the $129 \mathrm{~V}$ substrate and the type of generated amplified products. Indeed, np-dCJD prions were not amplified in our current cell-PMCA system irrespective of codon 129 genotype of the substrates, whereas $\mathrm{p}$-dCJD prions were consistently amplified with the $129 \mathrm{~V}$ substrates despite the PRNP codon 129 genotypic incompatibility between seed and substrate. Moreover, the amplified products from the p-dCJD prions with the $129 \mathrm{~V}$ substrates were similarly reactive with the $\operatorname{PrP}^{\mathrm{Sc}}$ type 2-specific antibody as those generated by sCJD-VV2 prions, despite the $\mathrm{PrP}^{\mathrm{Sc}}$ in the original p-dCJD homogenate being not reactive.

We previously reported that intermediate type $\operatorname{PrP}^{\mathrm{Sc}}$ with a downward size shift from type 1 and kuru plaques in p-dCJD were phenotypic features associated with the cross-sequential transmission of the V2 sCJD strain to individuals with the codon $129 \mathrm{MM}$ genotype. ${ }^{9-11,14}$ Hence, given the transmission properties of $\mathrm{p}$-dCJD prions, it is conceivable that $\mathrm{p}$-dCJD prions exhibit cell-PMCA amplification properties similar to those of other CJD subtypes associated with the V2 strain. Although the amplification factors varied among the $\mathrm{p}$-dCJD cases because of the variability in $\operatorname{PrP}^{\mathrm{Sc}}$ amount in the seeded brain homogenates, all p-dCJD cases were amplified up to a $10^{-3}$-fold dilution with the $129 \mathrm{~V}$ substrates.

To test the specificity of cell-PMCA as a new tool to differentiate between the acquired CJD derived from V2 strain and SCJD associated with M1 strain, we also performed cell-PMCA in two atypical CJD-129MM cases with plaques.
Although direct evidence of exposure to prion-contaminated brain tissues or surgical instruments was not obtained from the available clinical records of these patients, a transmission study has revealed that the two cases might actually be acquired CJD caused by infection with the V2 SCJD strain. ${ }^{16}$ In transmission experiments, the two atypical CJD cases exhibited the same properties as $\mathrm{p}$-dCJD prions. Consistent with the results of transmission studies, both atypical CJD cases showed amplification properties identical to those of $\mathrm{p}$-dCJD prions. These findings indicate that acquired CJD derived from the V2 strain can be easily identified by analyzing the characteristic amplification properties, ie, strong amplification with the $129 \mathrm{~V}$ substrate and generation of type 2 amplified products in cell-PMCA.

Transmission studies using knock-in humanized mice are a powerful tool to identify the causative origin of acquired prion diseases. ${ }^{11,16,26}$ However, they require an incubation period of over 2 years to yield significant results. Instead, cellPMCA will help us to rapidly find out acquired CJD associated with the V2 strain within just a week, even without definitive evidence of contact with prion-contaminated tissues.

Except for variant CJD prions, ${ }^{17,27}$ human prions have not been efficiently amplified by PMCA, ${ }^{27}$ whereas some animal prions such as those derived from hamster scrapie, ${ }^{28,29,30}$ bovine spongiform encephalopathy, ${ }^{31}$ or chronic wasting disease $^{32,33}$ can be efficiently amplified. The compatibility of the genotype at codon 129 between the seed and substrate is one of the most important factors for the efficient amplification of human prions. ${ }^{34}$ However, in the present study the amplification of the M1 or M2 CJD strains failed even with the matching $129 \mathrm{M}$ substrate. This might be due to inadequate PMCA buffer conditions $(50 \mathrm{mM}$ phosphate buffer ( $\mathrm{pH}$ 6.8), 1\% (v/v) Nonidet-P-40, 4 mM EDTA, $80 \mathrm{mM} \mathrm{NaCl}$ and complete PI), ineffective duration, or strength of sonication (five pulses of $5 \mathrm{~s}$ with 1-s rest) in our PMCA apparatus. Thus, although PMCA has not yet been put into practical use as an ultrasensitive assay for the detection of the whole spectrum of human prions, we propose cell-PMCA as a rapid diagnostic method for identifying acquired CJD associated with the V2 strain. To avoid the potential risk of CJD transmission via unidentified routes, further surveillance of acquired CJD cases using cell-PMCA, in addition to careful analyses of the phenotypic features of the patients, is needed.

Supplementary Information accompanies the paper on the Laboratory Investigation website (http://www.laboratoryinvestigation.org)

\section{ACKNOWLEDGMENTS}

This study was supported by Grant-in-Aid for Scientific Research from JSPS (AT:15K09307, TK: 25257506), Grants-in-Aid from the Ministry of Health, Labour and Welfare of Japan (AK), a grant from MEXT for the Joint Research Program of the Research Center for Zoonosis Control, Hokkaido University (TK), and a Grant-in-Aid for Scientific Research on Innovative Areas (Brain Protein Aging and Dementia Control) from MEXT (TK). Research for development of decontamination/disinfection procedures against various SCJD subgroups has 
been supported by a Grant-in-Aid from the Research Committee of Surveillance and Infection Control of Prion Disease, the Ministry of Health, Labor and Welfare of Japan (TK). We thank M Yamamoto for her excellent technical assistance and B Bell for critical review of the manuscript

\section{DISCLOSURE/CONFLICT OF INTEREST}

The authors declare no conflict of interest.

1. Parchi P, Giese A, Capellari S et al. Classification of sporadic Creutzfeldt-Jakob disease based on molecular and phenotypic analysis of 300 subjects. Ann Neurol 1999;46:224-233.

2. Brown P, Brandel JP, Sato T et al. latrogenic Creutzfeldt-Jakob Disease, final assessment. Emerg Infect Dis 2012;18:901-907.

3. Brown P, Brandel JP, Preece $M$ et al. latrogenic Creutzfeldt-Jakob disease: the waning of an era. Neurology 2006;67:389-393.

4. Hoshi K, Yoshino $\mathrm{H}$, Urata J et al. Creutzfeldt-Jakob disease associated with cadaveric dura mater grafts in Japan. Neurology 2000;55:718-721.

5. Kretzschmar HA, Sethi S, Foldvari Z et al. latrogenic Creutzfeldt-Jakob disease with florid plaques. Brain Pathol 2003;13:245-249.

6. Shimizu S, Hoshi K, Muramoto T et al. Creutzfeldt-Jakob disease with florid-type plaques after cadaveric dura mater grafting. Arch Neurol 1999;56:357-362.

7. Mochizuki Y, Mizutani T, Tajiri N et al. Creutzfeldt-Jakob disease with florid plaques after cadaveric dura mater graft. Neuropathology 2003:23:136-140.

8. Bishop MT, Will RG, Manson JC. Defining sporadic Creutzfeldt-Jakob disease strains and their transmission properties. Proc Natl Acad Sci USA 2010;107:12005-12010.

9. Kobayashi A, Asano M, Mohri S et al. Cross-sequence transmission of sporadic Creutzfeldt-Jakob disease creates a new prion strain. J Biol Chem 2007;282:30022-30028.

10. Kobayashi A, Sakuma N, Matsuura Y et al. Experimental verification of a traceback phenomenon in prion infection. J Virol 2010;84:3230-3238.

11. Kobayashi A, Asano M, Mohri S et al. A traceback phenomenon can reveal the origin of prion infection. Neuropathology 2009;29:619-624.

12. Noguchi-Shinohara M, Hamaguchi T, Kitamoto T et al. Clinical features and diagnosis of dura mater graft associated Creutzfeldt Jakob disease. Neurology 2007:69:360-367.

13. Yamada M, Noguchi-Shinohara M, Hamaguchi T et al. Dura mater graftassociated Creutzfeldt-Jakob disease in Japan: clinicopathological and molecular characterization of the two distinct subtypes. Neuropathology 2009;29:609-618.

14. Kobayashi A, Matsuura Y, Mohri S et al. Distinct origins of dura mater graft-associated Creutzfeldt-Jakob disease: past and future problems. Acta Neuropathol Commun 2014;2:32.

15. Nozaki I, Hamaguchi T, Sanjo N et al. Prospective 10-year surveillance of human prion diseases in Japan. Brain 2010;133:3043-3057.

16. Kobayashi A, Parchi P, Yamada $M$ et al. Transmission properties of atypical Creutzfeldt-Jakob disease: a clue to disease etiology? J Virol 2015;89:3939-3946.

17. Yokoyama $T$, Takeuchi $A$, Yamamoto $M$ et al. Heparin enhances the cell-protein misfolding cyclic amplification efficiency of variant Creutzfeldt-Jakob disease. Neurosci Lett 2011:498:119-123.
18. Kitamoto $\mathrm{T}$, Shin RW, Doh-ura $\mathrm{K}$ et al. Abnormal isoform of prion proteins accumulates in the synaptic structures of the central nervous system in patients with Creutzfeldt-Jakob disease. Am J Pathol 1992:140:1285-1294.

19. Kitamoto $T$, Ohta $M$, Doh-ura $K$ et al. Novel missense variants of prion protein in Creutzfeldt-Jakob disease or Gerstmann-Straussler syndrome. Biochem Biophys Res Commun 1993;191:709-714.

20. Notari S, Capellari S, Giese A et al. Effects of different experimental conditions on the $\operatorname{PrP}^{\mathrm{Sc}}$ core generated by protease digestion: implications for strain typing and molecular classification of CJD. J Biol Chem 2004;279:16797-16804.

21. Ishida C, Kakishima A, Okino S et al. Sporadic Creutzfeldt-Jakob disease with MM1-type prion protein and plaques. Neurology 2003;60: 514-517.

22. Schoene WC, Masters $\mathrm{CL}$, Gibbs $\mathrm{CJ}$ et al. Transmissible spongiform encephalopathy (Creutzfeldt-Jakob disease). Atypical clinical and pathological findings. Arch Neurol 1981;38:473-477.

23. Parchi $P$, Cescatti $M$, Notari $S$ et al. Agent strain variation in human prion disease: insights from a molecular and pathological review of the National Institutes of Health series of experimentally transmitted disease. Brain 2010;133:3030-3042.

24. Hizume M, Kobayashi A, Teruya K et al. Human prion protein (PrP) $219 \mathrm{~K}$ is converted to $\mathrm{PrP}^{\mathrm{SC}}$ but shows heterozygous inhibition in variant Creutzfeldt-Jakob disease infection. J Biol Chem 2009;284:3603-3609.

25. Grathwohl $\mathrm{KU}$, Horiuchi $\mathrm{M}$, Ishiguro $\mathrm{N}$ et al. Improvement of $\mathrm{PrP}^{\mathrm{SC}}$ detection in mouse spleen early at the preclinical stage of scrapie with collagenase-completed tissue homogenization and Sarkosyl- $\mathrm{NaCl}$ extraction of PrP ${ }^{\text {SC }}$. Arch Virol 1996;141:1863-1874.

26. Asano $M$, Mohri S, Ironside JW et al. vCJD prion acquires altered virulence through trans-species infection. Biochem Biophys Res Commun 2006;342:293-299.

27. McDowell KL, Nag N, Franco $Z$ et al. Blood reference materials from macaques infected with variant Creutzfeldt-Jakob disease agent. Transfusion 2015;55:405-412.

28. Soto C, Anderes L, Suardi S et al. Pre-symptomatic detection of prions by cyclic amplification of protein misfolding. FEBS Lett 2005;579: 638-642.

29. Castilla J, Saa P, Hetz C et al. In vitro generation of infectious scrapie prions. Cell 2005;121:195-206.

30. Saa P, Castilla J, Soto C. Ultra-efficient replication of infectious prions by automated protein misfolding cyclic amplification. J Biol Chem 2006;281:35245-35252.

31. Murayama $\mathrm{Y}$, Masujin $\mathrm{K}$, Imamura $\mathrm{M}$ et al. Ultrasensitive detection of $\operatorname{PrP}(\mathrm{Sc})$ in the cerebrospinal fluid and blood of macaques infected with bovine spongiform encephalopathy prion. J Gen Virol 2014;95: 2576-2588.

32. Kurt TD, Perrott MR, Wilusz CJ et al. Efficient in vitro amplification of chronic wasting disease PrP RES. J Virol 2007;81:9605-9608.

33. Johnson CJ, Aiken JM, McKenzie D et al. Highly efficient amplification of chronic wasting disease agent by protein misfolding cyclic amplification with beads (PMCAb). PLoS One 2012;7:e35383.

34. Jones $M$, Peden $A H$, Wight $D$ et al. Effects of human PrP ${ }^{S c}$ type and PRNP genotype in an in-vitro conversion assay. Neuroreport 2008;19: $1783-1786$ 